\title{
Weak module amenability for the second dual of a Banach algebra
}

\author{
M. Shabani Soltanmoradi, D. Ebrahimi Bagha, and \\ O. Pourbahri RAHPEYMA
}

\begin{abstract}
In this paper we study the weak module amenability of Banach algebras which are Banach modules over another Banach algebra with compatible actions. We show that for every module derivation $D$ : $A \longmapsto\left(\frac{\mathcal{A}}{J_{\mathcal{A}}}\right)^{*}$ if $D^{* *}\left(\mathcal{A}^{* *}\right) \subseteq W A P\left(\frac{\mathcal{A}}{J_{\mathcal{A}}}\right)$, then weak module amenability of $\mathcal{A}^{* *}$ implies that of $\mathcal{A}$. Also we prove that under certain conditions for the module derivation $D$, if $\mathcal{A}^{* *}$ is weak module amenable then $\mathcal{A}$ is also weak module amenable.
\end{abstract}

\section{Introduction}

The concept of amenability for Banach algebras was introduced by B. E. Johnson [13]. He showed that the group algebra $L^{1}(G)$ is amenable if and only if $G$ as a group is amenable. Subsequently, various generalizations of this notion - such as Banach modules - were studied by a number of authors ( see $[1,5,6,10,16]$ ). Amini [1] used this fact and developed the concept of module amenability for a Banach algebra $\mathcal{A}$ to the case where there is an extra $\mathfrak{A}$-module structure on $\mathcal{A}$. He showed that for an inverse semigroup $S$ with the set of idempotents $E, l^{1}(S)$ is $l^{1}(E)$-module amenable if and only if $S$ is amenable.

Let $\mathcal{A}$ be a Banach algebra and let $X$ be a Banach $\mathcal{A}$-module. A derivation $D: \mathcal{A} \longrightarrow X$ is a bounded linear operator such that, $D(a b)=a . D(b)+D(a) . b$ for all $a, b \in \mathcal{A}$. Also, a derivation $D$ is said to be inner if there exists $x \in X$ such that $D(a)=a . x-x . a$ for every $a \in A$.

A Banach algebra $\mathcal{A}$ is said to be amenable if every derivation from $\mathcal{A}$ into each dual Banach $\mathcal{A}$-module is inner [13].

Received April 9, 2021.

2020 Mathematics Subject Classification. 46H25, 43A07.

Key words and phrases. Module amenability, Banach algebra, the second module dual of a Banach algebra.

https://doi.org/10.12097/ACUTM.2021.25.19

Corresponding author: O. Pourbahri Rahpeyma 
A Banach algebra $\mathcal{A}$ is called weakly amenable [2] if every derivation $D$ : $\mathcal{A} \longrightarrow \mathcal{A}^{*}$ is inner. F. Gourdeau has shown that the amenability of $\mathcal{A}^{* *}$ implies the amenability of $\mathcal{A}[12]$. However, for weak amenability this result is not proved yet.

Problem: Let $\mathcal{A}^{* *}$ be a weakly amenable Banach algebra Can we conclude that $\mathcal{A}$ is also weakly amenable?

The above problem has been solved in certain settings. For example, in each of the following cases the above problem has a positive answer.

1 ) $\mathcal{A}$ is a left ideal in $\mathcal{A}^{* *}[11]$.

$2) \mathcal{A}$ is a dual Banach algebra $[4,10]$.

3 ) Every derivation $D: \mathcal{A} \longrightarrow \mathcal{A}^{*}$ satisfies $D^{* *}\left(\mathcal{A}^{* *}\right) \subseteq W A P(\mathcal{A})[9]$.

4 ) $\mathcal{A}$ is a right ideal of $\mathcal{A}^{* *}$ with $\mathcal{A}^{* *} \square \mathcal{A}=\mathcal{A}^{* *}[9]$.

In Section 2, we prove that if $\mathcal{A}$ is a Banach $\mathfrak{A}$-module and $J_{\mathcal{A}}$ is the closed ideal of $\mathcal{A}$ generated by $\{(a . \alpha) b-a(\alpha . b): a, b \in \mathcal{A}, \alpha \in \mathfrak{A}\}$ and every derivation $D: \mathcal{A} \longrightarrow\left(\frac{A}{J_{\mathcal{A}}}\right)^{*}$ satisfies $D^{* *}\left(\mathcal{A}^{* *}\right) \subseteq W A P\left(\frac{\mathcal{A}}{J_{\mathcal{A}}}\right)$, then weak module amenability of $\mathcal{A}^{* *}$ implies weak module amenability of $\mathcal{A}$. The case $\mathfrak{A}=\mathbb{C}$ yields our main theorem. In fact this result can be considered as an extension of [9, Theorem 2.1]. Also, we show that if $\mathcal{A}$ is a Banach $\mathfrak{A}$-module, such that $J_{\mathcal{A}}^{\perp \perp} \subseteq \mathcal{A}, \mathcal{A}^{* *} \cdot \frac{\mathcal{A}}{J_{\mathcal{A}}^{\perp} \perp}=\frac{\mathcal{A}^{* *}}{J_{\mathcal{A}}^{\perp} \perp}$ and $\frac{\mathcal{A}}{J_{\mathcal{A}}^{\frac{1}{}} \perp} \cdot \mathcal{A}^{* *} \subseteq \frac{\mathcal{A}}{J_{\mathcal{A}}^{\perp} \perp}$, then weak module amenability of $\mathcal{A}^{* *}$ implies weak module amenability of $\mathcal{A}$. This result can be considered as an extension of [9, Theorem 2.4].

\section{Main results}

Let $\mathcal{A}$ and $\mathfrak{A}$ be Banach algebras and let $\mathcal{A}$ be a Banach $\mathfrak{A}$-module such that

$$
(\alpha . a) b=\alpha .(a b),(a b) . \alpha=a(b . \alpha) \quad(a, b \in \mathcal{A}, \alpha \in \mathfrak{A}) .
$$

If $X$ is a Banach $\mathcal{A}$-module and a Banach $\mathfrak{A}$-module with compatible actions, such that

$$
\alpha .(a . x)=(\alpha . a) . x,(a . x) . \alpha=a .(x . \alpha) \quad(a \in \mathcal{A}, x \in X, \alpha \in \mathfrak{A}),
$$

and with similar operations for right actions, then $X$ is called an $\mathcal{A}$ - $\mathfrak{A}$-module. If, moreover,

$$
\alpha . x=x . \alpha \quad(\alpha \in \mathfrak{A}, x \in X),
$$

then $X$ is called a commutative $\mathcal{A}$ - $\mathfrak{A}$-module.

If $X$ is a ( commutative) Banach $\mathcal{A}$ - $\mathfrak{A}$-module so is $X^{*}$, with the following actions:

$$
\begin{aligned}
& \langle\alpha . f, x\rangle=\langle f, x . \alpha\rangle,\langle f . \alpha, x\rangle=\langle f, \alpha . x\rangle \\
& \langle a . f, x\rangle=\langle f, x . a\rangle,\langle f . a, x\rangle=\langle f, a . x\rangle \quad\left(a \in \mathcal{A}, x \in X, \alpha \in \mathfrak{A}, f \in X^{*}\right) .
\end{aligned}
$$


Let $X$ and $Y$ be $\mathcal{A}$-A-modules, and let $\phi: X \rightarrow Y$ satisfies the following conditions:

$$
\begin{aligned}
& \phi(\alpha . x)=\alpha . \phi(x), \phi(x . \alpha)=\phi(x) \cdot \alpha \\
& \phi(a . x)=a . \phi(x), \phi(x . a)=\phi(x) . a \quad(a \in \mathcal{A}, x \in X, \alpha \in \mathfrak{A}) .
\end{aligned}
$$

Then $\phi$ is called a module bihomomorphism.

Let $X$ be a commutative Banach $\mathcal{A}$ - $\mathfrak{A}$-module, then the projective tensor product $\mathcal{A} \hat{\otimes} X$ is an $\mathcal{A}$-A-module with the following actions:

$$
\begin{aligned}
& a .(b \otimes x)=(a b) \otimes x,(b \otimes x) \cdot a=b \otimes(x . a) \\
& \alpha .(b \otimes x)=(\alpha . b) \otimes x,(b \otimes x) \cdot \alpha=b \otimes(x . \alpha) \quad(a, b \in \mathcal{A}, x \in Y, \alpha \in \mathfrak{A}) .
\end{aligned}
$$

Now, define $\pi_{X}: \mathcal{A} \hat{\otimes} X \rightarrow X$ by

$$
\pi_{X}(a \otimes x)=a . x \quad(a \in \mathcal{A}, x \in X) .
$$

It is clear that $\pi_{X}$ is an $\mathcal{A}$-A-module bihomomorphism.

Let $I_{X}$ be the closed $\mathcal{A}$ - $\mathfrak{A}$-submodule of the projective tensor product $A \hat{\otimes} X$ generated by

$$
\{(a . \alpha) \otimes x-a \otimes(\alpha . x): a \in \mathcal{A}, \alpha \in \mathfrak{A}, x \in X\} .
$$

Also let $J_{X}$ be the closed submodule of $X$ generated by $\pi\left(I_{X}\right)$, that is,

$$
J_{X}=\overline{\left\langle\pi_{X}\left(I_{X}\right)\right\rangle}=\overline{\{(a . \alpha) \cdot x-a .(\alpha . x): a \in \mathcal{A}, \alpha \in \mathfrak{A}, x \in X\}} .
$$

In a particular case, when $X=\mathcal{A}, J_{\mathcal{A}}$ is the closed submodule in $\mathcal{A}$ generated by $\{(a . \alpha) b-a(\alpha . b)\}$ for $a, b \in \mathcal{A}$ and $\alpha \in \mathfrak{A}$.

Definition 1. The closed $\mathcal{A}$-A-modules $J_{X}{ }^{\perp}$ of $X^{*}$ and $J_{J_{X} \perp}^{\perp}$ of $X^{* *}$ are called the first and the second module dual of $X$, respectively.

In the case when $X$ is a commutative $\mathfrak{A}$-module, then $J_{X}^{\perp}=X^{*}$ and $J_{J_{X} \perp}^{\perp}=X^{* *}$.

Remark 1. Since $\left(\frac{\mathcal{A}}{J_{\mathcal{A}}}\right)^{*} \simeq J_{\mathcal{A}}{ }^{\perp}$, we have

$$
\left\langle\tilde{f}, a+J_{\mathcal{A}}\right\rangle=\langle f, a\rangle \quad(a \in A),
$$

when $f \in J_{\mathcal{A}}{ }^{\perp}$ is the corresponding element $\tilde{f} \in\left(\frac{\mathcal{A}}{J_{\mathcal{A}}}\right)^{*}$. Since $\left(\frac{\mathcal{A}}{J_{\mathcal{A}}}\right)^{* *} \simeq$ $\frac{A^{* *}}{J_{\mathcal{A}} \perp \perp}$, we have

$$
\langle\tilde{F}, \tilde{f}\rangle=\langle F, f\rangle \quad\left(\tilde{f} \simeq f \in J_{\mathcal{A}}{ }^{\perp}\right),
$$

where $F+J_{\mathcal{A}}{ }^{\perp \perp} \in \frac{A^{* *}}{J_{\mathcal{A}} \perp \perp}$ is the corresponding element $\tilde{F} \in\left(\frac{\mathcal{A}}{J_{\mathcal{A}}}\right)^{* *}$.

Note that $\left(\frac{\mathcal{A}}{J_{\mathcal{A}}}\right)^{*}$ is an $\mathcal{A}$-module, where the actions of $\mathcal{A}$ on $\left(\frac{\mathcal{A}}{J_{\mathcal{A}}}\right)^{*}$ are defined by:

$$
\left\langle\tilde{f} . a, b+J_{\mathcal{A}}\right\rangle=\left\langle\tilde{f}, a b+J_{\mathcal{A}}\right\rangle,\left\langle a . \tilde{f}, b+J_{\mathcal{A}}\right\rangle=\left\langle\tilde{f}, b a+J_{\mathcal{A}}\right\rangle \quad\left(a, b \in \mathcal{A}, \tilde{f} \in\left(\frac{\mathcal{A}}{J_{\mathcal{A}}}\right)^{*}\right) .
$$


Therefore the second module dual of $\mathcal{A}$ is a closed submodule of $\frac{\mathcal{A}^{* *}}{J_{\mathcal{A}}}$.

Definition 2. Let $\mathcal{A}$ and $\mathfrak{A}$ be two Banach algebras and $X$ be a Banach $\mathcal{A}$-A-module. A bounded linear map $D: \mathcal{A} \rightarrow X$ is a module derivation if $D$ satisfies the following relations:

$$
\begin{aligned}
D(a b) & =D(a) \cdot b+a \cdot D(b), \\
D(\alpha . a) & =\alpha \cdot D(a), D(a \cdot \alpha)=D(a) \cdot \alpha \quad(a, b \in \mathcal{A}, \alpha \in \mathfrak{A}) .
\end{aligned}
$$

Lemma 1. [15, Theorem 2.4] Let $X^{*}$ be a commutative Banach $\mathcal{A}-\mathfrak{A}$ module and $D: \mathcal{A} \rightarrow X^{*}$ be a module derivation, then $D(\mathcal{A}) \subseteq J_{X}{ }^{\perp}$.

Proof. For each $a, b \in \mathcal{A}, \alpha \in \mathfrak{A}$ and $x \in X$, we have $(a . \alpha) . x-a .(\alpha . x) \in$ $J_{X}$. Hence

$$
\langle D(b),(a . \alpha) \cdot x-a .(\alpha \cdot x)\rangle=\langle D(b) .(a . \alpha)-(D(b) \cdot a) . \alpha, x\rangle=0 .
$$

Motivated by Definition 2.1 and 2.3, the concept of module amenability was introduced and studied in $[1,2]$.

Definition 3. A Banach algebra $\mathcal{A}$ is called weakly module amenable (as an $\mathfrak{A}$-module) if $J_{\mathcal{A}}^{\perp}$ is a commutative $\mathfrak{A}$-module and each linear module derivation $D: \mathcal{A} \rightarrow J_{\mathcal{A}}{ }^{\perp}$ is inner.

Lemma 2. Let $\mathcal{A}$ be a Banach $\mathfrak{A}$-module, then $J_{J_{\mathcal{A}}^{\perp}}^{\perp}$ is a closed ideal of $\mathcal{A}^{* *}$.

Proof. Let $a \in \mathcal{A}, \alpha \in \mathfrak{A}, f \in J_{\mathcal{A}}^{\perp}$ and $F \in J_{J_{\mathcal{A}}{ }^{\perp}}^{\perp}$. If $a^{* *} \in \mathcal{A}^{* *}$, there exists a bounded net $a_{i}$ in $\mathcal{A}$ such that $w^{*}-\lim a_{i}=a^{* *}$. Since $J_{J_{\mathcal{A}}{ }^{\perp}}$ is an $\mathcal{A}$-module, $a_{i}((a . \alpha) f-a(\alpha . f)) \in J_{J_{\mathcal{A}}{ }^{\perp}}$ for each $i$. It follows that

$$
\begin{aligned}
\left\langle F \square a^{* *},(a . \alpha) f-a(\alpha . f)\right\rangle & =\left\langle F, a^{* *}((a . \alpha) f-a(\alpha . f))\right\rangle \\
& =\lim \left\langle F, a_{i}((a . \alpha) f-a(\alpha . f))\right\rangle=0 .
\end{aligned}
$$

Hence $F \square a^{* *} \in J_{J_{\mathcal{A}}}^{\perp}$. Similarly $a^{* *} \square F \in J_{J_{\mathcal{A}}}^{\perp}$.

Remark 2. If $\mathcal{A}$ is an $\mathfrak{A}$-module Banach algebra, then $J_{J_{\mathcal{A}} \perp}^{\perp} \cong \frac{\mathcal{A}^{* *}}{J_{\mathcal{A}}^{\perp \perp}}$.

Proof. Let $f \in J_{\mathcal{A}}^{\perp}$. Then

$$
\begin{aligned}
\langle(a . \alpha) f-a(\alpha . f), b\rangle & =\langle f, b(a . \alpha)\rangle-\langle\alpha \cdot f, b a\rangle \\
& =\langle f,(b a) \cdot \alpha\rangle-\langle f,(b a) \cdot \alpha\rangle=\langle f,(b a) \cdot \alpha-(b a) \cdot \alpha\rangle=0
\end{aligned}
$$

for every $a, b \in \mathcal{A}$ and $\alpha \in \mathfrak{A}$. Therefore, $(a . \alpha) f-a(\alpha . f)=0$.

Since $(a . \alpha) f-a(\alpha . f)$ is a basic member of $J_{J_{\mathcal{A}}}$, we have $J_{J_{\mathcal{A}}{ }^{\perp}}=0$. Thanks to lemma 2.6, $J_{J_{\mathcal{A}}^{\perp}}^{\perp}$ is a closed ideal in $\frac{\mathcal{A}^{* *}}{J_{\mathcal{A}}^{\perp \perp}}$, so that $J_{J_{\mathcal{A}}^{\perp}}^{\perp} \cong \frac{\mathcal{A}^{* *}}{J_{\mathcal{A}}^{\perp} \perp}$. 
For a Banach algebra $\mathcal{A}$, let $\frac{A}{J_{\mathcal{A}}}$ be a Banach $\mathcal{A}$-module whose left and right module actions are

and

$$
\pi_{1}: \mathcal{A} \times \frac{\mathcal{A}}{J_{\mathcal{A}}} \longrightarrow \frac{\mathcal{A}}{J_{\mathcal{A}}}, \quad \pi_{1}\left(a, b+J_{\mathcal{A}}\right)=a b+J_{\mathcal{A}}
$$

for $a, b \in \mathcal{A}$.

$$
\pi_{2}: \frac{\mathcal{A}}{J_{\mathcal{A}}} \times \mathcal{A} \longrightarrow \frac{\mathcal{A}}{J_{\mathcal{A}}}, \quad \pi_{2}\left(b+J_{\mathcal{A}}, \mathcal{A}\right)=b a+J_{\mathcal{A}}
$$

We denote $\frac{\mathcal{A}}{J_{\mathcal{A}}}$ with the above operations by $\left(\pi_{1}, \frac{\mathcal{A}}{J_{\mathcal{A}}}, \pi_{2}\right)$. Then $\left(\pi_{2}^{r * r}\right.$, $\left.\left(\frac{\mathcal{A}}{J_{\mathcal{A}}}\right)^{*}, \pi_{1}^{*}\right)$ is a Banach $\mathcal{A}$-module [8], which is called the dual of $\left(\pi_{1}, \frac{A}{J_{\mathcal{A}}}, \pi_{2}\right)$. Here $\pi_{2}^{r * r}: \mathcal{A} \times\left(\frac{\mathcal{A}}{J_{\mathcal{A}}}\right)^{*} \longrightarrow\left(\frac{\mathcal{A}}{J_{\mathcal{A}}}\right)^{*}$ and $\pi_{1}^{*}:\left(\frac{\mathcal{A}}{J_{\mathcal{A}}}\right)^{*} \times \mathcal{A} \longrightarrow\left(\frac{\mathcal{A}}{J_{\mathcal{A}}}\right)^{*}$ are given by $\pi_{2}^{r * r}(a, f)=a . f, \pi_{1}^{*}(f, a)=f . a \quad\left(a \in \mathcal{A}, f \in\left(\frac{\mathcal{A}}{J_{\mathcal{A}}}\right)^{*}\right)$. Since $\left(\pi_{2}^{r * r * * *}\right.$, $\left.\left(\frac{\mathcal{A}}{J_{\mathcal{A}}}\right)^{* * *}, \pi_{1}^{* * * *}\right)$ is the second dual of $\left(\pi_{2}^{r * r},\left(\frac{\mathcal{A}}{J_{\mathcal{A}}}\right)^{*}, \pi_{1}^{*}\right)$ (as a Banach $\mathcal{A}$-module), $\left(\frac{\mathcal{A}}{J_{\mathcal{A}}}\right)^{* * *}$ is an $\mathcal{A}^{* *}$-module.

Lemma 3. Let $\mathcal{A}$ be a Banach $\mathfrak{A}$-module, $X$ be a Banach $\mathcal{A}$-A-module and $D: \mathcal{A} \longrightarrow X$ be a module derivation. Then $D^{* *}: \mathcal{A}^{* *} \longrightarrow X^{* *}$ is a module derivation.

Proof. By [15, Lemma 2.9].

Remark 3. [15, Remark 3] Let $\mathcal{A}$ be a Banach algebra, then

$$
\left(J_{\mathcal{A}}^{\perp}\right)^{* *} \cong\left(\frac{\mathcal{A}}{J_{\mathcal{A}}}\right)^{* * *} \cong\left(\frac{\mathcal{A}^{* *}}{J_{\mathcal{A}}^{\perp \perp}}\right)^{*} .
$$

For a Banach algebra $\mathcal{A}$, we have

$$
\widehat{\left(\frac{\mathcal{A}}{J_{\mathcal{A}}}\right)^{*}} \cong \widehat{J_{\mathcal{A}}^{\perp}} \subseteq\left(\frac{\mathcal{A}^{* *}}{J_{\mathcal{A}^{* *}}}\right)^{*} \cong J_{\mathcal{A}^{* *}}^{\perp}
$$

Proof. If $f \in J_{\mathcal{A}}^{\perp}$, then $\left.f\right|_{J_{\mathcal{A}}}=0$. We will show that $\hat{f} \in J_{\mathcal{A}^{* *}}^{\perp}$. Take some $a^{* *} \in \mathcal{A}^{* *}$, let $a_{i}$ be a bounded net in $\mathcal{A}$ such that $w^{*}-\lim a_{i}=a^{* *}$, and let $a \in \mathcal{A}$ and $\alpha \in \mathfrak{A}$. Then

$$
\begin{aligned}
\left\langle\hat{f},(a . \alpha) a^{* *}-a\left(\alpha . a^{* *}\right)\right\rangle & =\left\langle(a . \alpha) a^{* *}-a\left(\alpha . a^{* *}\right), f\right\rangle \\
& =\lim _{i}\left\langle(a . \alpha) \hat{a_{i}}-a\left(\alpha . \hat{a_{i}}\right), f\right\rangle \\
& =\lim _{i}\left\langle(a . \alpha) \widehat{a_{i}-a}\left(\alpha . a_{i}\right), f\right\rangle \\
& =\lim _{i}\left\langle f,(a . \alpha) a_{i}-a\left(\alpha . a_{i}\right)\right\rangle=0 .
\end{aligned}
$$

Since $\hat{f}$ is linear and continuous, $\hat{f} \in J_{\mathcal{A}^{* *}}^{\perp}$.

Definition 4. The collection of all $f \in J_{\mathcal{A}}^{\perp}$ such that $a^{* *}+J_{\mathcal{A}}^{\perp \perp} \longmapsto$ $\left\langle b^{* *} \square a^{* *}+J_{\mathcal{A}}^{\perp \perp}, f\right\rangle$ is $w^{*}$-continuous on $J_{J_{\mathcal{A}} \perp}^{\perp}$ for every $b^{* *}+J_{\mathcal{A}}^{\perp \perp} \in J_{J_{\mathcal{A}} \perp}^{\perp}$ is denoted by $W A P\left(\frac{\mathcal{A}}{J_{\mathcal{A}}}\right)$. 
Definition 5. Let $\mathcal{A}$ be a Banach $\mathfrak{A}$-module, then $\mathcal{A}$ is called ideal Arens regular if $m \square n=m \diamond n$ for any $m, n \in J_{J_{\mathcal{A}}}^{\perp}$.

Remark 4. If $\mathcal{A}$ is a commutative $\mathfrak{A}$-module then $J_{\mathcal{A}}^{\perp \perp}=0$. Therefore $\mathcal{A}^{* *}=J_{J_{\mathcal{A}} \perp}^{\perp}$. This shows that our definition is compatible with the original definition of Arens regularity [3].

Now we are ready to state the first main result of this section.

Theorem 1. Let $\mathcal{A}$ be a Banach $\mathfrak{A}$-module and $\mathcal{A}^{* *}$ be weak module amenable. For every module derivation $D: \mathcal{A} \longrightarrow\left(\frac{\mathcal{A}}{J_{\mathcal{A}}}\right)^{*}$ if $D^{* *}\left(\mathcal{A}^{* *}\right) \subseteq$ $W A P\left(\frac{\mathcal{A}}{J_{\mathcal{A}}}\right)$, then $\mathcal{A}$ is weak module amenable.

Proof. Let $\varphi: \frac{\mathcal{A}}{J_{\mathcal{A}}} \longrightarrow \frac{\mathcal{A}^{* *}}{J_{\mathcal{A}}^{\perp \perp}}$ be defined by $\varphi\left(a+J_{\mathcal{A}}\right)=\hat{a}+J_{\mathcal{A}}^{\perp \perp}$ for each $a \in \mathcal{A}$. We prove that $\varphi$ is well-defined. Let $a_{1}+J_{\mathcal{A}}=a_{2}+J_{\mathcal{A}}$ for $a_{1}+J_{\mathcal{A}}$, $a_{2}+J_{\mathcal{A}} \in \frac{A}{J_{\mathcal{A}}}$, then we have $a_{1}-a_{2} \in J_{\mathcal{A}}$. Therefore, for each $f \in J_{\mathcal{A}}^{\perp}$ we have

$$
\left\langle\widehat{a_{1}-a_{2}}, f\right\rangle=\left\langle f, a_{1}-a_{2}\right\rangle=0
$$

thus $\widehat{a_{1}-a_{2}} \in J_{\mathcal{A}}^{\perp \perp}$. Hence $\hat{a_{1}}+J_{\mathcal{A}}^{\perp \perp}=\hat{a_{2}}+J_{\mathcal{A}}^{\perp \perp}$. Now let $D: \mathcal{A} \longrightarrow J_{\mathcal{A}}^{\perp}$ be a module derivation. By Remark 3, we may assume that $\varphi^{*} \circ D^{* *}$ is a map from $\mathcal{A}^{* *}$ to $\left(\frac{\mathcal{A}^{* *}}{J_{\mathcal{A}^{* *}}}\right)^{*}$. Hence, we have to show that $\varphi^{*} \circ D^{* *}$ is a module derivation. By Lemma $3, D^{* *}: \mathcal{A}^{* *} \longrightarrow\left(J_{\mathcal{A}}^{\perp}\right)^{* *}$ is a module derivation, i.e.,

$$
D^{* *}\left(a^{* *} \square b^{* *}\right)=\pi_{1}^{* * * *}\left(D^{* *}\left(a^{* *}\right), b^{* *}\right)+\pi_{2}^{r * r * * *}\left(a^{* *}, D^{* *}\left(b^{* *}\right)\right)
$$

for every $a^{* *}, b^{* *} \in \mathcal{A}$. Hence for each $a^{* *}, b^{* *} \in \mathcal{A}$,

$$
\varphi^{*} \circ D^{* *}\left(a^{* *} \square b^{* *}\right)=\varphi^{*}\left(\pi_{1}^{* * * *}\left(D^{* *}\left(a^{* *}\right), b^{* *}\right)\right)+\varphi^{*}\left(\pi_{2}^{r * r * * *}\left(a^{* *}, D^{* *}\left(b^{* *}\right)\right) .\right.
$$

Let $a_{i}, b_{j}$ be bounded nets in $\mathcal{A}$ such that $w^{*}-\lim _{i} a_{i}=a^{* *}$ and $w^{*}$ $\lim _{j} b_{j}=b^{* *}$. For each $a+J_{\mathcal{A}} \in \frac{\mathcal{A}}{J_{\mathcal{A}}}$, we have

$$
\begin{aligned}
\left\langle\varphi^{*}\left(\pi_{2}^{r * r * * *}\left(a^{* *}, D^{* *}\left(b^{* *}\right)\right)\right), a+J_{\mathcal{A}}\right\rangle & =\left\langle\pi_{2}^{r * r * * *}\left(a^{* *}, D^{* *}\left(b^{* *}\right)\right), \varphi\left(a+J_{\mathcal{A}}\right)\right\rangle \\
& =\lim _{i} \lim _{j}\left\langle\pi_{2}^{r * r}\left(\hat{a_{i}}, D^{* *}\left(\hat{b_{j}}\right)\right), \hat{a}+J_{\mathcal{A}}^{\perp}\right\rangle \\
& =\lim _{i} \lim _{j}\left\langle D^{* *}\left(\hat{b_{j}}\right), \pi_{2}\left(\hat{a}+J_{\mathcal{A}}^{\perp \perp}, \hat{a_{i}}\right)\right\rangle \\
& =\lim _{i} \lim _{j}\left\langle D^{* *}\left(\hat{b_{j}}\right), \widehat{a a_{i}}+J_{\mathcal{A}}^{\perp}\right\rangle \\
& =\lim _{i} \lim _{j}\left\langle D^{* *}\left(\hat{b_{j}}\right), \varphi\left(\pi_{2}\left(a+J_{\mathcal{A}}, a_{i}\right)\right)\right\rangle \\
& =\lim _{i} \lim _{j}\left\langle\varphi^{*}\left(D^{* *}\left(\hat{b_{j}}\right)\right), \pi_{2}^{r}\left(a_{i}, a+J_{\mathcal{A}}\right)\right\rangle \\
& =\lim _{i} \lim _{j}\left\langle\pi_{2}^{r * r}\left(a_{i}, \varphi^{*}\left(D^{* *}\left(\hat{b_{j}}\right)\right)\right), a+J_{\mathcal{A}}\right\rangle \\
& =\left\langle\pi_{2}^{r * r * * *}\left(a^{* *}, \varphi^{*}\left(D^{* *}\left(b^{* *}\right)\right), a+J_{\mathcal{A}}\right\rangle .\right.
\end{aligned}
$$


Therefore,

$$
\varphi^{*}\left(\pi_{2}^{r * * * * *}\left(a^{* *}, D^{* *}\left(b^{* *}\right)\right)\right)=\pi_{2}^{r * r * * *}\left(a^{* *}, \varphi^{*}\left(D^{* *}\left(b^{* *}\right)\right)\right) .
$$

Also

$$
\begin{aligned}
\left\langle\varphi^{*}\left(\pi_{1}^{* * * *}\left(D^{* *}\left(a^{* *}\right), b^{* *}\right)\right), a+J_{\mathcal{A}}\right\rangle & =\left\langle\pi_{1}^{* * * *}\left(D^{* *}\left(a^{* *}\right), b^{* *}\right), \hat{a}+J_{\mathcal{A}}^{\perp \perp}\right\rangle \\
& =\lim _{j} \lim _{i}\left\langle\pi_{1}^{*}\left(D\left(\hat{a_{i}}\right), \hat{b_{j}}\right), \hat{a}+J_{\mathcal{A}}^{\perp \perp}\right\rangle \\
& =\lim _{j} \lim _{i}\left\langle D\left(\hat{a_{i}}\right), \pi_{1}\left(\hat{b_{j}}, \hat{a}+J_{\mathcal{A}}^{\perp \perp}\right)\right\rangle \\
& =\lim _{j} \lim _{i}\left\langle D\left(\hat{a_{i}}\right), \widehat{b_{j} a}+J_{\mathcal{A}}^{\perp \perp}\right\rangle \\
& =\lim _{j} \lim _{i}\left\langle D\left(\hat{a}_{i}\right), \varphi\left(b_{j} a+J_{\mathcal{A}}\right)\right\rangle \\
& =\lim _{j}\left\langle D^{* *}\left(a^{* *}\right), \varphi\left(b_{j} a+J_{\mathcal{A}}\right)\right\rangle \\
& =\left\langle D^{* *}\left(a^{* *}\right), \varphi\left(b^{* *} a+J_{\mathcal{A}}\right)\right\rangle \\
& =\left\langle D^{* *}\left(a^{* *}\right), \varphi\left(\pi_{1}^{* * *}\left(b^{* *}, a+J_{\mathcal{A}}\right)\right)\right\rangle \\
& =\left\langle\varphi^{*}\left(D^{* *}\left(a^{* *}\right)\right), \pi_{1}^{* * *}\left(b^{* *}, a+J_{\mathcal{A}}\right)\right\rangle \\
& \left.=\left\langle\pi_{1}^{* * * *}\left(\varphi^{*}\left(D^{* *}\left(a^{* *}\right)\right), b^{* *}\right)\right), a+J_{\mathcal{A}}\right\rangle .
\end{aligned}
$$

Therefore,

$$
\varphi^{*}\left(\pi_{1}^{* * * *}\left(D^{* *}\left(a^{* *}\right), b^{* *}\right)\right)=\pi_{1}^{* * * *}\left(\varphi^{*}\left(D^{* *}\left(a^{* *}\right), b^{* *}\right)\right) .
$$

Hence $\varphi^{*} \circ D^{* *}$ is a derivation. For each $\alpha \in \mathfrak{A}$ and $a^{* *} \in \mathcal{A}^{* *}$, we have

$$
\varphi^{*} \circ D^{* *}\left(\alpha \cdot a^{* *}\right)=\alpha \cdot \varphi^{*} \circ D^{* *}\left(a^{* *}\right), \quad \varphi^{*} \circ D^{* *}\left(a^{* *} \cdot \alpha\right)=\varphi^{*} \circ D^{* *}\left(a^{* *}\right) \cdot \alpha \text {. }
$$

Thus $\varphi^{*} \circ D^{* *}: \mathcal{A}^{* *} \longrightarrow\left(\frac{\mathcal{A}^{* *}}{J_{\mathcal{A}^{* *}}}\right)^{*}$ is a module derivation.

As $\mathcal{A}^{* *}$ is weakly module amenable, there exist $F \in\left(\frac{\mathcal{A}^{* *}}{J_{\mathcal{A}^{* *}}}\right)^{*}$ such that $\varphi^{*} \circ D^{* *}=\delta_{F}$. Let $f=\varphi^{*}(F) \in\left(\frac{\mathcal{A}}{J_{\mathcal{A}}}\right)^{*}$. It follows that $D=\delta_{f}$; i.e $D$ is inner. Therefore $\mathcal{A}$ is weakly module amenable.

Corollary 1. Let $\mathcal{A}$ be Banach $\mathfrak{A}$-module which is ideal Arens regular. Suppose that every module derivation from $\mathcal{A}$ to $\left(\frac{\mathcal{A}}{J_{\mathcal{A}}}\right)^{*}$ is weakly compact. If $\mathcal{A}^{* *}$ is weakly module amenable, then so is $\mathcal{A}$.

Proof. Since $\mathcal{A}$ is ideal Arens regular, we have $W A P\left(\frac{\mathcal{A}}{J_{\mathcal{A}}}\right)=\left(\frac{\mathcal{A}}{J_{\mathcal{A}}}\right)^{*}$. Let $D: \mathcal{A} \longrightarrow\left(\frac{\mathcal{A}}{J_{\mathcal{A}}}\right)^{*}$ be a module derivation. Since $D$ is weakly compact, $D^{* *}\left(\mathcal{A}^{* *}\right) \subseteq\left(\frac{\mathcal{A}}{J_{\mathcal{A}}}\right)^{*}=W A P\left(\frac{\mathcal{A}}{J_{\mathcal{A}}}\right)$. By Theorem 1 , the weak module amenability of $\mathcal{A}^{* *}$ implies that of $\mathcal{A}$.

Corollary 2. [9, Corollary 2.1] Let $\mathcal{A}$ be Arens regular and suppose that every derivation from $\mathcal{A}$ to $\mathcal{A}^{*}$ is weakly compact. If $\mathcal{A}^{* *}$ is weakly amenable, then so is $\mathcal{A}$. 
Proof. Take $\mathfrak{A}=\mathbb{C}$ in Corollary 1.

We are ready to state the second main result of this section.

Theorem 2. Let $\mathcal{A}$ be a Banach $\mathfrak{A}$-module such that $J_{\mathcal{A}}^{\perp \perp} \subseteq \mathcal{A}, \mathcal{A}^{* *} \cdot \frac{\mathcal{A}}{J_{A}^{\perp \perp}}=$ $\frac{\mathcal{A}^{* *}}{J_{\mathcal{A}}^{\perp \perp}}$ and $\frac{\mathcal{A}}{J_{\mathcal{A}}^{\perp \perp}} . \mathcal{A}^{* *} \subseteq \frac{\mathcal{A}}{J_{\mathcal{A}}^{\perp \perp}}$. If $\mathcal{A}^{* *}$ is weakly module amenable, then so is $\mathcal{A}$.

Proof. Let $\varphi: \frac{\mathcal{A}}{J_{\mathcal{A}}} \longrightarrow \frac{\mathcal{A}^{* *}}{J_{\mathcal{A}}^{\perp \perp}}$ be defined by $\varphi\left(a+J_{\mathcal{A}}\right)=\hat{a}+J_{\mathcal{A}}^{\perp \perp}$ for each $a \in \mathcal{A}$. Applying the same argument that was used in the proof of Theorem 1 , we see that $\varphi$ is well defined. We prove that $\varphi^{*} \circ D^{* *}: A^{* *} \longrightarrow\left(\frac{\mathcal{A}^{* *}}{J_{\mathcal{A}^{* *}}}\right)^{*}$ is a module derivation.

Let $D: \mathcal{A} \longrightarrow J_{\mathcal{A}}^{\perp}$ be a module derivation. By Lemma $3, D^{* *}: \mathcal{A}^{* *} \longrightarrow$ $\left(J_{\mathcal{A}}^{\perp}\right)^{* *}$ is a module derivation, i.e.

$D^{* *}\left(a^{* *} \square b^{* *}\right)=\pi_{1}^{* * * *}\left(D^{* *}\left(a^{* *}\right), b^{* *}\right)+\pi_{2}^{r * r * * *}\left(a^{* *}, D^{* *}\left(b^{* *}\right)\right)\left(a^{* *}, b^{* *} \in \mathcal{A}\right)$.

Thus, for each $a^{* *}, b^{* *} \in \mathcal{A}$,

$$
\varphi^{*} \circ D^{* *}\left(a^{* *} \square b^{* *}\right)=\varphi^{*}\left(\pi_{1}^{* * *}\left(D^{* *}\left(a^{* *}\right), b^{* *}\right)\right)+\varphi^{*}\left(\pi_{2}^{r * r * * *}\left(a^{* *}, D^{* *}\left(b^{* *}\right)\right)\right.
$$

By the proof of Theorem 1, we obtain

$$
\varphi^{*}\left(\pi_{2}^{r * r * * *}\left(a^{* *}, D^{* *}\left(b^{* *}\right)\right)\right)=\pi_{2}^{r * r * * *}\left(a^{* *}, \varphi^{*}\left(D^{* *}\left(b^{* *}\right)\right)\right) .
$$

For proving the second part of the equation (1), we have

$$
\begin{aligned}
\left\langle\varphi^{*}\left(\pi_{1}^{* * * *}\left(D^{* *}\left(a^{* *}\right), b^{* *}\right), a+J_{\mathcal{A}}\right\rangle\right. & =\left\langle\pi_{1}^{* * * *}\left(D^{* *}\left(a^{* *}\right), b^{* *}\right), \varphi\left(a+J_{\mathcal{A}}\right)\right\rangle \\
& =\left\langle\pi_{1}^{* * * *}\left(D^{* *}\left(a^{* *}\right), b^{* *}\right), \hat{a}+J_{\mathcal{A}}^{\perp \perp}\right\rangle \\
& =\left\langle D^{* *}\left(a^{* *}\right), \pi_{1}^{* * *}\left(b^{* *}, \hat{a}+J_{\mathcal{A}}^{\perp \perp}\right)\right\rangle .
\end{aligned}
$$

We claim that

$$
\pi_{1}^{* * *}\left(b^{* *}, \hat{a}+J_{\mathcal{A}}^{\perp \perp}\right)=\varphi\left(\pi_{1}^{* * *}\left(b^{* *}, \hat{a}+J_{\mathcal{A}}^{\perp \perp}\right)\right) .
$$

For each $a \in \mathcal{A}$,

$$
\begin{aligned}
\left\langle D^{* *}\left(a^{* *}\right), \pi_{1}^{* * *}\left(b^{* *}, \hat{a}+J_{\mathcal{A}}^{\perp \perp}\right)\right\rangle & =\left\langle D^{* *}\left(a^{* *}\right), \varphi\left(\pi_{1}^{* * *}\left(b^{* *}, \hat{a}+J_{\mathcal{\mathcal { A }}}^{\perp \perp}\right)\right)\right\rangle \\
& \left.=\left\langle\varphi^{*}\left(D^{* *}\left(a^{* *}\right)\right), \pi_{1}^{* * *}\left(b^{* *}, \hat{a}+J_{\mathcal{A}}^{\perp \perp}\right)\right)\right\rangle \\
& \left.=\left\langle\pi_{1}^{* * * *} \circ \varphi^{*}\left(D^{* *}\left(a^{* *}\right), b^{* *}\right)\right), \hat{a}+J_{\mathcal{A}}^{\perp}\right\rangle .
\end{aligned}
$$

This shows that

$$
\varphi^{*}\left(\pi_{1}^{* * * *}\left(D^{* *}\left(a^{* *}\right), b^{* *}\right)\right)=\pi_{1}^{* * * *}\left(\varphi^{*}\left(D^{* *}\left(a^{* *}\right), b^{* *}\right)\right) .
$$

Proof of the claim (2):

Let $f \in J_{\mathcal{A}}^{\perp}$. Since $\hat{a}+J_{\mathcal{A}}^{\perp \perp} \in \frac{\mathcal{A}^{* *}}{J_{\mathcal{A}}^{\perp \perp}}$, there exist members $d^{* *} \in \mathcal{A}^{* *}$ and $c \in \mathcal{A}$ such that $\hat{a}+J_{\mathcal{A}}^{\perp \perp}=d^{* *} \square c+J_{\mathcal{A}}^{\perp \perp}$ and $c \square \hat{a}+J_{\mathcal{A}}^{\perp \perp} \in \frac{\mathcal{A}}{J_{\mathcal{A}}^{\perp}}$, hence

$$
\left\langle\pi_{1}^{* * *}\left(b^{* *}, \hat{a}+J_{\mathcal{A}}^{\perp \perp}\right), f\right\rangle=\left\langle\pi_{1}^{* * *}\left(b^{* *}, d^{* *} \square c+J_{\mathcal{A}}^{\perp \perp}\right), f\right\rangle
$$




$$
\begin{aligned}
& =\lim _{i} \lim _{j}\left\langle\pi_{1}\left(\hat{b}_{i}, \hat{d}_{j} \square c+J_{\mathcal{A}}^{\perp \perp}\right), f\right\rangle \\
& =\lim _{i} \lim _{j}\left\langle\widehat{b_{i} d_{j} c}+J_{\mathcal{A}}^{\perp \perp}, f\right\rangle \\
& =\lim _{i} \lim _{j}\left\langle\left(b_{i} d_{j} c+J_{\mathcal{A}}\right), \varphi^{*}(f)\right\rangle \\
& =\lim _{i} \lim _{j}\left\langle\varphi^{*}(f), b_{i} d_{j} c+J_{\mathcal{A}}\right\rangle .
\end{aligned}
$$

On the other hand, we have

$$
\begin{aligned}
\left\langle\varphi\left(\pi_{1}^{* * *}\left(b^{* *}, \hat{a}+J_{\mathcal{A}}^{\perp \perp}\right), f\right\rangle\right. & =\left\langle\pi_{1}^{* * *}\left(b^{* *}, \hat{a}+J_{\mathcal{A}}^{\perp \perp}, \varphi^{*}(f)\right\rangle\right. \\
& =\left\langle\pi_{1}^{* * *}\left(b^{* *}, d^{* *} \square c+J_{\mathcal{A}}^{\perp}, \varphi^{*}(f)\right\rangle\right. \\
& =\lim _{i} \lim _{j}\left\langle\pi_{1}\left(\hat{b_{i}}, \hat{d}_{j} \square c+J_{\mathcal{A}}^{\perp \perp}\right), \varphi^{*}(f)\right\rangle \\
& =\lim _{i} \lim _{j}\left\langle\widehat{b_{i} d_{j} c}+J_{\mathcal{A}}^{\perp \perp}, \varphi^{*}(f)\right\rangle \\
& =\lim _{i} \lim _{j}\left\langle b_{i} \widehat{d_{j} c+} J_{\mathcal{A}}, \varphi^{*}(f)\right\rangle \\
& =\lim _{i} \lim _{j}\left\langle\varphi^{*}(f), b_{i} d_{j} c+J_{\mathcal{A}}\right\rangle .
\end{aligned}
$$

Hence

$$
\pi_{1}^{* * *}\left(b^{* *}, \hat{a}+J_{\mathcal{A}}^{\perp \perp}\right)=\varphi\left(\pi_{1}^{* * *}\left(b^{* *}, \hat{a}+J_{\mathcal{A}}^{\perp \perp}\right)\right) .
$$

Corollary 3. [9, Theorem 2.4] Let $\mathcal{A}$ be a right ideal in $\mathcal{A}^{* *}$ and suppose that $\mathcal{A}^{* *} \square \mathcal{A}=\mathcal{A}^{* *}$. If $\mathcal{A}^{* *}$ is weakly amenable, then $\mathcal{A}$ is weakly amenable.

Proof. Take $\mathfrak{A}=\mathbb{C}$ in Theorem 2.

\section{Acknowledgements}

The authors are grateful for the reviewer's valuable comments that improved the manuscript.

\section{References}

[1] M. Amini, Module amenability for semigroup algebras, Semigroup Forum 69 (2004), $302-312$.

[2] M. Amini and A. Bodaghi, Module amenability and weak module amenability for second dual of Banach algebras, Cham. J. Math. 2 (2010), 57-71.

[3] R. Arens, The adjoint of a bilinear operation, Proc. Amer. Math. Soc. 2 (1951), 839848.

[4] S. Barootkoob and H. R. Ebrahimi Vishki, Lifting derivations and n-weak amenability of the second dual of a Banach algebra, Bull. Aust. Math. Soc. 83 (2011), 122-129.

[5] J. B. Conway, A course in Functional Analysis, Springer-Verlag, New York, 1985.

[6] H. G. Dales, Banach Algebra and Automatic Continuity, Clarendon, Oxford, 2000. 
[7] H. G. Dales, F. Ghahramani, and N. Grønbæek, Derivations into iterated dual of Banach spaces, Studia Math. 128 (1998), 19-54.

[8] H. G. Dales, A. Rodrigues-Palacios, and M. V. Velasco, The second transpose of a derivation, J. London Math. Soc. 64 (2001), 707-721.

[9] M. Eshaghi Gordji and M. Filali, Weak amenability of the second dual of a Banach algebra, Studia Math. 182 (2007), 205-213.

[10] F. Ghahramani and J. Laali, Amenability and topological center of the second dual of Banach algebras, Bull. Austral. Soc. 85 (2002), 191-197.

[11] F. Ghahramani, R. J. Loy, and G. A. Willis, Amenability and weak amenability of the second conjugate Banach algebras, Proc. Amer. Math. Soc. 124 (1996), 1489-1497.

[12] F. Gourdeau, Amenability and the second dual of a Banach algebra, Studia Math. 125 (1997), 75-81.

[13] B. E. Johnson, Cohomology in Banach algebra, Memoirs Amer. Math. Soc. 127 (1972).

[14] S. Mohammadzadeh and H. R. E. Vishki, Arens regularity of module actions and the second adjoint of a derivation, Bull. Aust. Math. Soc. 77 (2008), 465-476.

[15] O. Pourbahri Rahpeyma, A. Kamel Mirmostafaee, and D. Ebrahimi Bagha, Weak module amenability of module dual Banach algebra, Honam Math. ( to appear )

[16] V. Runde, Lectures on Amenability, Lecture Notes in Mathematics, 1774. Springer, Berlin, 2002.

Department of Mathematics, Faculty of Science, Central Tehran Branch,, ISLAMIC AZAD University, TEHRAN, IRAN.

E-mail address: mehrdad_sh554@yahoo.com

E-mail address: e_bagha@yahoo.com

Department of Mathematics, Faculty of Science, Chalous Branch,, Islamic

Azad University, Chalous, Iran.

E-mail address: omidpourbahri@yahoo.com 